Nota científica

(Short communication)

\title{
PRIMER REGISTRO DE ACALITUS ORTHOMERA (KEIFER) (ACARI: ERIOPHYIDAE) ASOCIADO AL CULTIVO DE LA ZARZAMORA (RUBUS FRUTICOSUS VAR. TUPY) EN ZIRACUARETIRO, MICHOACÁN, MÉXICO
}

\section{NEW RECORD OF ACALITUS ORTHOMERA (KEIFER) (ACARI: ERIOPHYIDAE) IN BLACKBERRY (RUBUS FRUTICOSUS VAR. TUPY) IN ZIRACUARETIRO, MICHOACÁN, MEXICO}

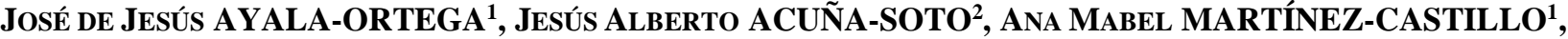 \\ M. B. N. LARA-CHÁVEZ ${ }^{3}$, MARGARITA VARGAS-SANDOVAL ${ }^{4 *}$

\begin{abstract}
${ }^{1}$ Instituto de Investigaciones Agropecuarias y Forestales, Universidad Michoacana de San Nicolás de Hidalgo. Km. 9.5 Carretera Morelia-Zinapecuaro, Tarímbaro. Michoacán C.P. 58880, México.<cordoba_1821@ @otmail.com>; <mabel_66@hotmail.com> ${ }^{2}$ Colegio de Postgraduados. Km 36.5 Carretera México-Texcoco, Montecillo, Texcoco, Estado de México C.P. 56230, México. <coleoptero77@hotmail.com>

${ }^{3}$ Facultad de Agrobiología "Presidente Juárez", Universidad Michoacana de San Nicolás de Hidalgo. <chavez12001@yahoo.com.mx> Baja Ciudad Universitaria C.P. 58030, Morelia, Michoacán. <vargasmarga@hotmail.com>
\end{abstract} \\ ${ }^{4}$ Facultad de Biología. Universidad Michoacana de San Nicolás de Hidalgo. Avenida Francisco J. Mujica S/N Edificio "R" Planta \\ *Autor de correspondencia: <vargasmarga@hotmail.com>
}

Recibido: 25/05/2018; aceptado: 07/10/2019; publicado en línea: 30/10/2019

Editor responsable: Gabriel Villegas Guzmán

Ayala-Ortega, J. J., Acuña-Soto, J. A., Martínez-Castillo, A. M., Lara-Chávez, M. B. N., VargasSandoval, M. (2019) Primer registro de Acalitus Orthomera (Keifer) (Acari: Eriophyidae) asociado al cultivo de la zarzamora (Rubus fruticosus var. tupy) en Ziracuaretiro, Michoacán, México. Acta Zoológica Mexicana (nueva serie), 35, 1-4. https://doi.org/10.21829/azm.2019.3501223

RESUMEN. Acalitus orthomera (Keifer) es registrado por primera vez en el Municipio de Ziracuaretiro, Michoacán, México, asociado a brotes vegetativos en cultivos de zarzamora; se proporciona información sobre su daño y su importancia en este cultivo es discutida.

Ayala-Ortega, J. J., Acuña-Soto, J. A., Martínez-Castillo, A. M., Lara-Chávez, M. B. N., VargasSandoval, M. (2019) New record of Acalitus orthomera (Keifer) (Acari: Eriophyidae) in blackberry (Rubus fruticosus var. tupy) in Ziracuaretiro, Michoacán, Mexico. Acta Zoológica Mexicana (nueva serie), 35, 1-4. https://doi.org/10.21829/azm.2019.3501223

ABSTRACT. The eriophyid mite Acalitus orthomera (Keifer, 1951) is record for the first time in the state of Michoacán, Mexico, associated to vegetative shoots in cultured blackberry; data are provided on its damage and its importance in this crop as discussed.

El 85\% de las especies de la superfamilia Eriophyoidea corresponden a la familia Eriophyidae, con más de 4,000 especies descritas (de Lillo \& Skoracka, 2010). Dentro de esta familia destaca el género Acalitus con 
97 especies descritas en el mundo (Amrine \& Stasny, 1994). En 1970, Wilson reportó a A. batissimus en México y más tarde García-Valencia y Hoffmann (1997) describieron a A. santibanezi; sin embargo, en el país los eriofioideos han sido poco estudiados y por el momento se desconoce el número total de especies presentes (Acuña-Soto et al., 2010).

Acalitus orthomera (Keifer) ha sido registrada en Estados Unidos de América, Nueva Zelanda y Polonia (Amrine \& Stasny, 1994) asociada con zarzamoras cultivadas; en estas plantas provoca ampollas dentro de los brotes, agallas en los peciolos que retrasan o limitan el desarrollo de ramas y de los mismos, y generan ampollas en la base de las drupas (Baker et al., 1996); sin embargo, a pesar de estos daños, este eriófido no ha sido considerado de importancia económica para el cultivo. Recientemente en Brasil fue asociado a los frutos, pero su relación con el descoloramiento de las drupas no es clara (Trinidad et al., 2108). Por otra parte, las especies A. essigi (Hassan) y Phyllocoptes gracilis (Nalepa) si están directamente relacionadas con el daño en el fruto y en infestaciones severas lo secan, mermando su calidad, lo que ocasiona pérdidas en su comercialización (Davies et al., 2001; Pye \& de Llilo, 2010).

En el mes de mayo del 2016, en el huerto denominado "Santa Marcela" ubicado en el municipio de Ziracuaretiro, fueron recolectados brotes tiernos y frutos de zarzamora Rubus fruticosus var. tupy, los cuales se colocaron en bolsas de polietileno con cierre hermético. Se llevaron al laboratorio de Acarología de la Facultad de Agrobiología "Presidente Juárez" de la Universidad Michoacana de San Nicolás de Hidalgo. Los ácaros se extrajeron, preservaron y montaron de acuerdo con lo propuesto por Amrine y Manson (1996). La determinación taxonómica a nivel de género se basó en Amrine et al. (2003). La confirmación específica del eriófido encontrado se realizó empleando la descripción original realizada por Keifer (1951). Las imágenes de los especímenes fueron tomadas con microscopía diferencial de contraste de interferencia (DIC) y los trabajos morfométricos fueron realizados tomando fotografías en microscopía de contraste de fases. Las imágenes se analizaron mediante el programa Image Tool 3,0 (Wilcox et al., 2002). Las medidas se presentan en $\mu \mathrm{m}$ y son el promedio del total de ejemplares; las máximas y mínimas están entre paréntesis. Los ejemplares obtenidos fueron depositados en la colección de referencia de plagas agrícolas de la Facultad de Agrobiología "Presidente Juárez".

Material examinado: Acalitus orthomera $(n=15)$, Michoacán, Ziracuaretiro, huerto "Santa Marcela"; $19^{\circ}$ 24' 50.22' $\mathrm{N},-101^{\circ} 55^{\prime} 12.60$ ' O; 1,317 msnm; VI-2016; 15 + ex Rubus fruticosus var. tupy, (brotes vegetativos); Col. Ayala-Ortega.

Acalitus orthomera (Keifer, 1951)

Diagnosis: Hembra. Cuerpo. Vermiforme de 192 (180-200) de largo por 37 (35-40) de ancho. Gnatosoma. Pequeño y curveado hacia debajo, de 20 (18-21) de largo. Escudo prodorsal (Fig. 1). Con forma subtriangular, de 29 (27-31) de ancho y 23 (21-25) de largo, con un diseño de líneas distribuidas de la siguiente manera: línea media presente, líneas admedias divergiendo posteriormente, primera línea lateral orientada hacia atrás y bifurcándose delante de los tubérculos dorsales; las líneas se hacen menos evidentes lateralmente debido a numerosas líneas cortas que se intercalan con las líneas centrales; lateralmente presenta una prominente mancha "ocelar". Tubérculos dorsales en el margen posterior del escudo y separados por 17.5 (15.5-19.5); sedas sc de 20 (18-23) de largo y proyectadas hacia atrás. Patas I. De 23 (23-25) de largo; tibia sin la seda $b v$, y de 6 (4-8) de largo; solenidio de 6 (4-7); empodio de cuatro ramas. Patas II. De 21 (19-23) de largo; tibia de 2.5 (2-3.5); tarso de 5 (4-7); solenido de 7.5 (5.5-9.5). Región coxigenital. Con uno o dos anillos incompletos y uno a dos anillos completos. Platos coxisternales. Totalmente microtuberculadas, las coxas anteriores unidas hacia el margen anterior. Apodemas prosternal apenas visible. Opistosoma. Con 68 (65-70) anillos completamente microtuberculados, los microtubérculos son pequeños y redondeados; seda lateral $c 2$ de 21 (20-22) de largo, en el anillo 10 (9-11) desde el escudo prodorsal; seda $d$ de 35 (32-36), en el anillo 22 (20-24); seda $e$ de 26 (24-27), en el anillo 41 (39-43); seda $f$ de 12 (11-14), en el anillo 5 (4-6) desde el final del opistosoma; sedas accesorias $h l$ aparentemente ausentes. Apodema genital. Son anteriormente acuminados. Genitalia externa. De 18 (17-20) de ancho y 
de 10 (9-11) de largo, la placa que la cubre, presenta de 10 (9-11) costillas irregulares y longitudinales; seda $3 a$ de 11 (9-12) de largo (Fig. 1).

Macho. No encontrado.

Observaciones de campo: En este estudio las poblaciones de A. orthomera fueron bajas, de entre 10 y 15 ácaros por yema, sin observarse daño como lo reportado en otras partes del mundo (Baker et al., 1996). Por su parte, Rebollar-Alviter et al. (2013) la reportan en yemas vegetativas necrosadas, indicando que no hay una correlación directa entre el daño y la presencia de A. orthomera en Michoacán.
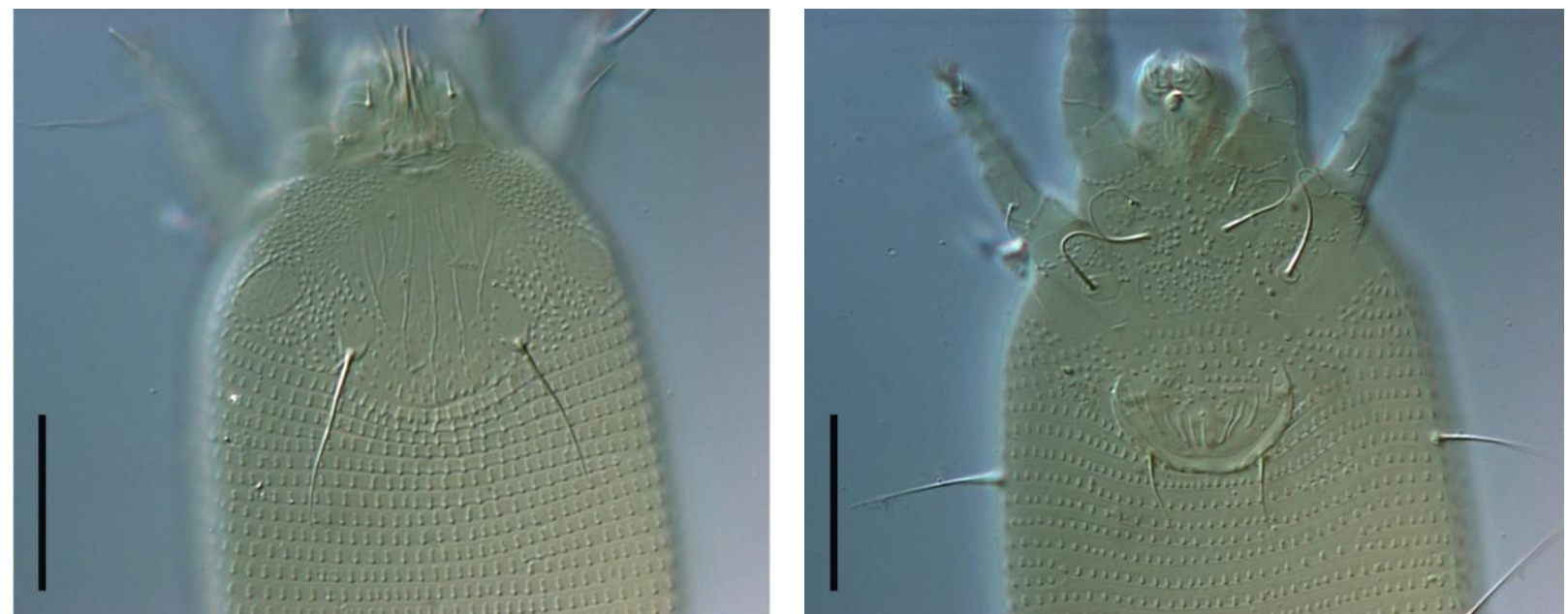

Figura 1. Acalitus orthomera (Keifer, 1951), Derecha. Detalle del escudo donde se aprecia el diseño de líneas que lo conforman, así como las prominentes manchas "ocelares". Izquierda. Detalle de la región coxigenital, donde se aprecia el diseño de la placa que cubre la genitalia. Barra de escala $20 \mu \mathrm{m}$.

Se ha reportado otra especie de Acalitus pero asociada al fruto (Lemus-Soriano et al., 2016); estos autores mencionan que los daños ocasionados pueden causar pérdidas del 60 al 100\% en la producción de la frutilla, pero no profundiza en la taxonomía de la especie. Es posible que se trate de A. essigi (Hassan), la cual Lemus-Soriano (2017) menciona que está presente en la región productora de zarzamora en Michoacán desde el 2012. En el presente estudio no se encontraron eriófidos en el fruto y es probable que A. essigi haya sido determinada erróneamente, cuestión que se hace irresoluble al no tener el material para su corroboración y, por el momento, su presencia en el estado es dudosa.

Agradecimientos. A Rogelio Rubio Maldonado, propietario de las huertas de Ziracuaretiro, por permitirnos realizar las colectas. A la Coordinación de la Investigación Científica, UMSNH por el apoyo al proyecto "Estudio de los ácaros plaga y depredadores en el cultivo de la zarzamora en los municipios de Taretan y Ziracuaretiro", a CONACyT por la beca de los estudios de maestría del primer autor. A la Bióloga Guadalupe López Campos y el Dr. Ignacio M. Vázquez Rojas por el apoyo para la toma de las fotografías en el laboratorio de acarología "Anita Hoffman" de la Facultad de Ciencias de la UNAM.

\section{LITERATURA CITADA}

Acuña-Soto, J. A., Estrada-Venegas, E. G., Equihua-Martínez, A. (2010) Catalog of eriophyoid mites (Prostigmata: Eriophyoidea) of México. Pp. 4. En: G. J. de Moraes, R. C. Castilho, C. H. W. Flechtmann (Eds.). The XIII International Congress of Acarology. Recife, Pernambuco, Brazil. Abstracts Book. 
Amrine, W. J., Stansny, T. H. A., Fletchtman, H. W. C. (2003) Revised keys to world genera of Eriophyoidea (Acari: Prostigmata). Indira. Publishing House. West Bloomfield, Michigan, 244 pp.

Amrine Jr., J. A., Manson, D. C. M. (1996) Preparation, Mounting and Descriptive Study of Eriophyid Mites. Pp. 383-396. En: E. E. Lindquist, M. W. Sabelis, J. Bruin (Eds.) Eriophyoid mites - Their biology, natural enemies and control. El Sevier Science Publ. Amsterdam, The Netherlands.

Amrine Jr., J. A., Stasny, T. A. (1994) Catalog of the Eriophyoidae (Acarina: Prostigmata) of the world. West Bloomfield, Michigan, USA. Indira Publishing House, $198 \mathrm{pp}$.

Baker, E. W., Kono, T., Amrine Jr, J. W., Delfinado-Baker, M., Stasny, T. A. (1996) Eriophyoid mites of the United States. Indira Publishing House, W. Bloomfield, MI, 394 pp.

Hoffman, A., López-Campos, M. G. (2000) Biodiversidad de ácaros de México. UNAM. CONABIO, 230 $\mathrm{pp}$.

Davies, J. T., Allen, G. R., Willians, M. A. (2001) Intraplant distribution of Acalitus essigi (Acari: Eriophyoidea) on blackberries (Rubus fruticosus agg.). Experimental and Applied Acarology, 25, 625-639. https://doi.org/10.1023/A:1016179817089

de Lillo, E., Skoracka, A. (2010) What's “cool” on eriophyoid mites? Pp 3-30. En: E. D. Uekermann (Ed.). Eriophyoid mites: progress and prognoses. New York (NY), Springer.

García-Valencia, A. S., Hoffmann, A. (1997) Especie nueva de acaro eriófido en México (Prostigmata: Eriophyidae). Anales del Instituto de Biología, serie Zoología, 68 (2), 253-260. http://revistas.unam.mx/index.php/zoo/article/view/7189/6695

Keifer, H. H. (1951) Eriophyid Studies XVII. Bulletin of the Department of Agriculture, State of California, 40, 93-104.

Lemus-Soriano, B. A. (2017) El Ácaro del berry rojo, una amenaza en la producción de zarzamora. Serie Frutillas Núm. 16. Artículos Técnicos de INTAGRI. México, 2 pp. https://www.intagri.com/articulos/frutillas/el-acaro-del-berry-rojo

Lemus-Soriano, B. A., Pérez-Aguilar, D. A., Garza-Blanco, J. (2016) Insecticidas biorracionales para el control de Acalitus sp. (Prostigmata: Eriophyidae) en zarzamora. Entomología mexicana, 3, $316-31$. http://www.entomologia.socmexent.org/revista/entomologia/2016/EA/Em\%20316-319.pdf

Pye, D. R. L., de Lillo, E. (2010) A review of the eriophyoid mites (Acari: Eriophyoidea) on Rubus spp. in Britain, with a new species (Diptilomiopidae) and two new records. Zootaxa, 2677, 15-26. http://www.mapress.com/zootaxa/2010/f/z02677p026f.pdf

Rebollar-Alviter, A., Otero-Colina, G., Calderón-Zavala, G., López-Medina, J., Sánchez-García, P., Flores-Martínez, B. A., López-Cruz, I. (2013) El papel de los ácaros eriófidos en la necrosis de yemas florales de zarzamora (Rubus spp.). En Memorias del XV Congreso Nacional de la Sociedad Mexicana de Ciencias Hortícolas A. C. Del 10 al 13 de septiembre, Puebla, Puebla, México.

Trinidad, O. T. C., Duarte, E. M., Silva da Cunha, E., Navia, D. (2018). Eriophyoid mites associated with the blackberry in Brazil - a new species in the genus Diptacus Keifer 1951 (Diptilomiopidae) and first report and supplementary description of Acalitus orthomerus (Keifer, 1951) (Eriophyidae). Systematic \& Applied Acarology, 23 (6), 1199-1216. http://doi.org/10.11158/saa.23.6.15.

Wilson, N. S. (1970) Some Eriophyid species (Acarina: Eriophyidae) from Mexico. Annals of the Entomological Society of America, 63 (4), 1125-1136. https://doi.org/10.1093/aesa/63.4.1125.

Wilcox, D., Dove, B., Doss, M., Greer, D. (2002) UTHSCSA. Image Tool for Windows, Version 3.00. University of Texas, Health Science Center in San Antonio. 